\title{
Sichtbar oder unsichtbar?
}

\section{Vom Kampf um Aufmerksamkeit in der visuellen Kultur}

\section{Von Markus Schroer}

Zusammenfassung: Unter visueller Kultur wird in diesem Beitrag nicht nur verstanden, dass wir immer mehr visuelles Material konsumieren, sondern es auch in steigendem Maße selbst produzieren. Der zeitdiagnostische Diskurs um die visuelle Kultur zeigt eine Privilegierung des Sichtbaren und die Bedeutungszunahme von Bildern auf allen Ebenen der (post)modernen Gesellschaft an. Alles was Bedeutung erlangen will, muss zum Bild werden. Dabei gilt: Wer oder was es versteht, auf sich aufmerksam zu machen, wird sichtbar. Wer oder was es nicht versteht, Aufmerksamkeit auf sich zu lenken, bleibt unsichtbar. Aufmerksamkeit zu erzielen und Sichtbarkeit zu erlangen kann in einer visuellen Kultur als identisch behandelt werden. Mit Hilfe eines analytischen Aufmerksamkeits-Schemas, das zwischen vier verschiedenen Daseinsmöglichkeiten in der visuellen Kultur zu unterschieden erlaubt, soll aufgezeigt werden, dass die Stellung und Position von Akteuren zunehmend davon abhängt, ob sie sich sichtbar oder unsichtbar machen können oder ob sie von anderen sichtbar bzw. unsichtbar gemacht werden.

Angesichts der enormen Fülle an visuellem Material, das uns täglich umgibt, ist kaum mehr länger zu bestreiten, dass wir in einer ,visuellen Kultur“ (Jenks 1995; Mirzoeff 1999; Mitchell 2008 b) leben. Nicht nur wenn wir ein Museum betreten, ins Kino gehen, durch das Fernsehprogramm zappen oder im Internet surfen, stößt unser Blick auf Bilder, Fotos und Filme. Auch jenseits dieser privilegierten Orte des Bildkonsums sind wir auf Schritt und Tritt der Konfrontation mit bewegten wie unbewegten Bildern ausgesetzt. In den eigenen Wohnräumen, im Wartezimmer des Arztes, in den Lounges am Flughafen, in U-Bahnstationen und Bahnhofshallen, Restaurants, Banken, Reisebüros, Shopping Malls und Fußballstadien, in Geschäften, Einkaufszentren und Bars, an zahlreichen Arbeitsplätzen, an Gebäudefassaden, ja sogar in Fahrstühlen und Bedürfnisanstalten - an all diesen Orten versorgen uns nahezu allgegenwärtige Bildschirme kontinuierlich mit Bildern. Durch die ebenso große Ubiquität von Kameras - auf die wir in Parkgaragen, öffentlichen Plätzen, Krankenhäusern, Kitas, Schulen, privaten Hauseingängen, Schwimmbädern und vielen anderen Orten stoßen - geraten wir dabei auch selbst in den Fokus der „Sehmaschinen“(Virilio 1989), die uns mit unserem eigenen medialen Bild konfrontieren. Wir sind damit nicht mehr nur Rezipienten von diversem Bildmaterial, sondern werden auch selbst zunehmend zum Objekt von Bildern. Damit nicht genug, sind wir in einem steigenden Ausmaß auch zu Bildproduzenten avanciert, die sich an der allgemeinen Verbreitung und Vermehrung der Bilder aktiv beteiligen. Spätestens durch die enorme Ausbreitung der Digitalfotografie, der Fotohandys und Homepages stehen inzwischen nicht mehr wenige Bildproduzenten vielen Bildkonsumenten gegenüber. Die Verbreitung der visuellen Technologien hat vielmehr zur Auflösung dieser starren Einteilung geführt, sodass wir heute immer schon zugleich Bildproduzenten und Bildkonsumenten, sowohl Sender als auch Empfänger von Bildern sind. Erst diese Entwicklung liefert m.E. die Berechtigung dazu, von einer visuellen Kultur zu sprechen. Visuelle Kultur bedeutet dabei vor allem die Privilegierung des Sichtbaren und die Bedeutungszunahme von Bildern auf allen Ebenen der (post)modernen Gesellschaft (vgl. Denzin 1995; Burri 2008; Raab 2008). Alles, was Bedeutung erlangen will, muss zum Bild werden. Dabei gilt: Wer oder was es versteht, auf sich aufmerksam zu machen, wird sichtbar. Wer oder was es nicht versteht, Aufmerksamkeit auf sich zu lenken, bleibt unsichtbar. Aufmerksamkeit zu erzielen und Sichtbarkeit zu erlangen kann in einer visuellen Kultur als identisch behandelt werden. In dieser von Bildern dominierten Kultur wachsen einerseits die Möglichkeiten, die Blicke auf sich zu ziehen, weil die Technologien und Medien 
dafür bereit und vielen zur Verfügung stehen. Doch je mehr Menschen von diesen Möglichkeiten Gebrauch machen, desto geringer wird andererseits die Chance, sichtbar zu werden, weil man im Meer der Bilder unterzugehen droht. Vor diesem Hintergrund wird ein verbissener „Kampf um (die) Aufmerksamkeit“ geführt (Münch 1995: 83; Rötzer 1998: 87ff; Franck 1998: 14; Nolte 2005; Schroer 2007). Den Zusammenhang von Selbstdarstellung, Sichtbarkeit / Unsichtbarkeit, Kampf um Aufmerksamkeit und visueller Kultur gilt es im Folgenden näher aufzuschlüsseln: Dabei wird in einem ersten Schritt der ebenso vielfach verwendete wie nur selten näher bestimmte Begriff der Aufmerksamkeit vorgestellt (1). Anschließend wird gezeigt, dass wir nicht deshalb in einer visuellen Kultur leben, weil wir einem ständig wachsenden Vorrat an Bildern ausgesetzt, sondern in steigendem Ausmaß an der Bildproduktion beteiligt sind (2). In einem dritten Argumentationsschritt geht es darum zu zeigen, dass der Kampf um Aufmerksamkeit in einer visuellen Kultur als Kampf um Sichtbarkeit ausgetragen wird (3). Mit Hilfe eines analytischen Aufmerksamkeits-Schemas, das zwischen vier verschiedenen Daseinsmöglichkeiten in der visuellen Kultur zu unterschieden erlaubt, soll abschließend gezeigt werden, dass für menschliche und andere Akteure viel davon abhängt, ob sie sich sichtbar oder unsichtbar machen können oder ob sie von anderen sichtbar bzw. unsichtbar gemacht werden (4).

\section{Was ist Aufmerksamkeit? Annäherung an einen vieldeutigen Begriff}

Aufmerksamkeit ist ein ebenso weit verbreiteter wie vieldeutiger Begriff. In der Psychologie, der Philosophie und der Pädagogik wird man ebenso fündig wie in der Neurophysiologie und in den Medienwissenschaften. Doch während er etwa in der Psychologie durchaus als fest institutionalisiertes Forschungsfeld gelten kann (vgl. Ribot 1908; Neumann 1971), fehlt es der Soziologie bis heute an einer systematischen Auseinandersetzung mit dem Begriff Aufmerksamkeit. Zwar finden sich quer durch die soziologische Theoriengeschichte Einträge zur Aufmerksamkeit, die von Emile Durkheim und Georg Simmel über Alfred Schütz, Max Scheler und Erving Goffman bis zu Niklas Luhmann, Zygmunt Bauman und Bruno Latour reichen. Eine eingehende Untersuchung aber, die die Aufmerksamkeit in den Mittelpunkt der Anstrengungen rücken würde, ist bis heute ausgeblieben. Gilt schon für die Philosophie, dass sie in der Aufmerksamkeit „kein Thema erster Ordnung“ (Blumenberg 2004: 182) gesehen hat, so trifft dies für die Soziologie in noch weit größerem Ausmaß zu. In den meisten Fällen kommt der Begriff vor, ohne dass er einer genaueren Klärung und Erläuterung unterzogen würde. Es scheint sich von selbst zu verstehen, was mit Aufmerksamkeit gemeint ist, jeder kann sich etwas darunter vorstellen. Will man es bei der Einschätzung nicht belassen, dass ,Jedermann

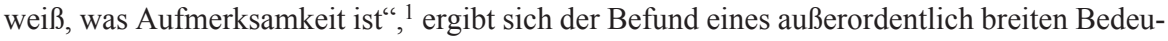
tungsspektrums, das hier nicht einmal im Ansatz nachvollzogen werden kann (vgl. dazu Neumann 1971; Crary 2002). Durch die wechselvolle Geschichte der Aufmerksamkeitsforschung hindurch aber lässt sich eine immer wiederkehrende Fragestellung ausmachen, die für den vorliegenden Zusammenhang von zentraler Bedeutung ist: Warum nehmen wir etwas Bestimmtes wahr und anderes - das wir ebenso hätten wahrnehmen können - nicht (vgl. Waldenfels 2004: 228)? Schon angesichts dieser Frage stoßen wir in dem breit gefächerten Aufmerksamkeitsdiskurs auf eine Fülle von Antworten, aus denen sich jedoch zwei grundlegende Auffassungen herausfiltern lassen.

1 Diese Aussage stammt von William James, der es auch selbst nicht dabei belassen hat, sondern fortfährt: „Es handelt sich um das geistige Inbesitznehmen eines von scheinbar mehreren gleichzeitig möglichen Objekten oder Gedankengängen in klarer und lebendiger Form. Zu ihrem Wesen gehört das wie auf einen Brennpunkt sich richtende Konzentrieren des Bewußtseins. Dazu gehört ferner die Abkehr von Objekten, um sich wirksam mit anderen zu beschäftigen." (William James zitiert nach Norman 1973: 19). 
Auf der einen Seite haben wir es mit Theorien der Aufmerksamkeit zu tun, die Aufmerksamkeit als Willensakt bzw. als Tätigkeit auffassen. Diesem Verständnis zufolge weist der Aufmerksamkeitsbegriff eine große Nähe zu den Begriffen Interesse und Konzentration auf etwa bei Wilhelm Wundt, William James (vgl. Kohn 1999) und George Herbert Mead. ${ }^{2}$ Aufmerksamkeit wird als die gezielte und freiwillige Hinwendung eines Subjekts auf eine bestimmte Sache definiert. Auf der anderen Seite gibt es ein Verständnis von Aufmerksamkeit, das nicht der Steuerung eines Subjekts unterliegt, sondern sich als eigenmächtig erweist. Hier wird davon ausgegangen, dass Phänomene, Ereignisse, Begebenheiten und Informationen sich jenseits eines Willensaktes dem Bewusstsein aufdrängen. Nach dieser Lesart steht es dem Subjekt gerade nicht frei, sich einer Sache zuzuwenden oder sie zu ignorieren. Vielmehr wird unterstellt, dass es Reize gibt, die so gravierend, einprägsam oder auffällig sind, dass die Aufmerksamkeit sich diesem einfach zuwenden muss - unabhängig vom Willen des Subjekts.

Es ist unübersehbar, dass das erste Verständnis von Aufmerksamkeit im Einklang mit dem Bild eines souveränen Subjekts und einem bürgerlichen Bildungsideal steht, nach dem sich ein Individuum im Laufe eines komplexen Sozialisations- und Erziehungsprozesses verschiedenen Themen und Tätigkeiten zuwendet und dabei die Fähigkeit erlernt, sich auf die jeweiligen Gegenstandsbereiche ernsthaft zu konzentrieren, ohne sich vom eingeschlagenen Weg abbringen und von den im Alltag stets lauernden Ablenkungen verführen zu lassen. Aufmerksamkeit wird hier zu einer Tugend, deren Aufbau mit disziplinarischen Mitteln zu erreichen versucht wird (vgl. Crary 2002; Ribot 1908).

Das zweite Verständnis von Aufmerksamkeit geht von einem leicht zu beeinflussenden Individuum aus, das gerade nicht seinen eigenen Impulsen und Interessen folgen kann, sondern von einer immer lauter, bunter und greller werdenden Umwelt geradezu gezwungen wird, aufzumerken. Die moderne Kultur mit ihren Tanzlokalen, Amüsiermeilen und Lichtspieltheatern, mit ihrer Begeisterung für Autorennen, das Boxen und den Fußball, ihrer Orientierung an Bewegung, Beschleunigung und Mobilität (vgl. Maase 1997; Gumbrecht 2001; Geisthövel / Knoch 2005), ihrer Suche nach dem Außergewöhnlichen, dem noch nie da gewesenen, der Sensation (vgl. Türcke 2002), erscheint nach dieser Lesart als eine Überwältigungskultur, die dem Einzelnen nicht länger die Entscheidung darüber überlässt, was oder wem er seine Aufmerksamkeit schenken will und was oder wem eben nicht. An diese Auffassung von Aufmerksamkeit haben eine Fülle von kulturkritischen Stimmen anknüpfen können, welche die Besorgnis teilen, dass das moderne Individuum zum willenlosen Spielball gesellschaftlicher Kräfte und kultureller Einflüsse degeneriert.

Schon diese beiden grundsätzlichen Auffassungen, die in der einschlägigen Literatur unter dem Namen „willkürliche / unwillkürliche“ bzw. ,aktive / passive Aufmerksamkeit“ (vgl.

2 „Das menschliche Wesen ist ein aufmerksames Wesen; seine Aufmerksamkeit kann sich bestimmten Reizen zuwenden, die relativ schwach sind. Wir können entfernte Geräusche aufnehmen. Unser ganzer Intelligenzprozeß scheint in der Aufmerksamkeit zu gründen, durch die bestimmte Reize ausgewählt werden. Andere Reize, die auf das System einhämmern, werden irgendwie abgelenkt. Wir wenden unsere Aufmerksamkeit einer bestimmten Sache zu. Nicht nur öffnen wir uns bestimmten Reizen und verschließen uns anderen, sondern unsere Aufmerksamkeit ist ebenso ein organisierender Prozeß wie ein selektiver. [...] Unsere Aufmerksamkeit ermöglicht es uns, jenen Bereich zu organisieren, in dem wir handeln werden. Hier haben wir den Organismus als einen, der handelt und seine Umwelt bestimmt. Er besteht nicht nur aus einer Anzahl passiver Sinnesorgane, auf die die von außen kommenden Reize einwirken, sondern er wendet sich nach außen und bestimmt, worauf er reagieren wird, organisiert also diese Welt.“ (Mead 1968: 64). 
Münsterberg 1996; Ribot 1908; Kohn 1999) ${ }^{3}$ geführt werden, lassen deutlich werden, dass im Aufmerksamkeitsdiskurs nichts weniger verhandelt wird als die Frage nach dem grundsätzlichen Verständnis und Stellenwert des modernen Individuums. Zur Debatte steht, ob das Individuum als ein autonomes Individuum beschrieben werden kann oder als ein beliebig manipulierbares Individuum gelten muss. Der Begriff der unwillkürlichen bzw. passiven Aufmerksamkeit resultiert aus der Erschütterung über die Verführbarkeit des menschlichen Bewusstseins, das sich nicht - den eigenen Interessen folgend - der Umwelt zuwendet, sondern offenbar von dieser gelenkt zu werden vermag. Wäre es jedoch so, dass es gar keiner äußeren Impulse bedürfte, um die Aufmerksamkeit des Individuums zu wecken, weil dieses allein seinen eigenen Intentionen folgt, wenn es diesem oder jenem seine Aufmerksamkeit schenkt, wären alle Versuche der äußeren Einflussnahme zum Scheitern verurteilt. Wäre es dagegen so, dass das Individuum nicht zumindest ein Mitspracherecht daran hat, welchem Ereignis oder welchem Phänomen, welcher Information oder welcher Person es seine Aufmerksamkeit schenken will und welchen nicht, dann würde der Kampf um Aufmerksamkeit erst gar nicht geführt werden müssen, in den die Akteure der postmodernen Kommunikationsgesellschaft jedoch unverkennbar verstrickt sind. Nur weil sie zwar prinzipiell verführbar, nicht aber beliebig manipulierbar sind, kommt der Kampf um Aufmerksamkeit überhaupt zustande. Das Subjekt ist also weder als so souverän zu denken, wie es der Begriff der willkürlichen Aufmerksamkeit nahe legt, noch als so determiniert, wie es der Begriff der unwillkürlichen Aufmerksamkeit suggeriert. ${ }^{4}$

Von diesen beiden unterschiedlichen Möglichkeiten, Aufmerksamkeit zu denken, ist eine dritte Lesart von Aufmerksamkeit zu unterscheiden, die nicht der Frage folgt, warum sich das Subjekt gerade diesen und keinen anderen Objekten zuwendet und welche Instanz diese Aufmerksamkeitsaufwendung bewirkt, sondern das Empfangen von Aufmerksamkeit für die eigene Person und deren Handlungen in den Mittelpunkt rückt. Bei dieser Form der Aufmerksamkeit handelt es sich keineswegs um eine Erfindung der Mediengesellschaft, wie die aktuelle Literatur suggeriert (vgl. Franck 1998; Beck / Schweiger 2001; Bleicher / Hickethier 2002; Pörksen / Krischke 2010; Bublitz 2010), sondern um eine unverzichtbare Voraussetzung für Sozialität. Wer ganz und gar ohne Zuwendung anderer Menschen bleibt, ist schlicht nicht überlebensfähig. Deshalb geht es im Folgenden um die Frage, mit welchen Mitteln der Selbstdarstellung es den verschiedensten sozialen Akteuren gelingt, die Aufmerksamkeit der anderen auf sich zu lenken. Angesichts der visuellen Kultur, in der wir leben, wird der in diesem Zusammenhang aktuell viel diskutierte „Kampf um Aufmerksamkeit“ als ein Kampf um Sichtbarkeit ausgetragen.

3 Die Unterscheidung geht offenbar auf den Physiker und Philosophen Gustav Theodor Fechner zurück, auch wenn sie bereits bei Descartes angelegt ist (vgl. Neumann 1971: 635ff). Bei Max Scheler (1954: 164) findet sich die Unterscheidung einer „aktive(n) oder passive(n) Aufmerksamkeit“, bei Alfred Schütz und Thomas Luckmann (2003: 258ff) die von „freiwilliger Zuwendung“ und ,erzwungener Aufmerksamkeit" bei ähnlichem Bedeutungsgehalt. Entgegen der kategorialen Trennung beider Formen sehen sie nur "graduelle Unterschiede“ zwischen ihnen und kommen damit der hier verfolgten Perspektive entgegen. $\mathrm{Zu}$ den verschiedenen Formen der Aufmerksamkeit vgl. auch Waldenfels (2004: 261ff).

4 Bedenkt man, dass Ribot (1908), der eine natürliche und absichtslose von einer künstlichen und willkürlichen Aufmerksamkeit unterscheidet, letztere als „Produkt der Zivilisation“ (Ribot 1908: 50) ansieht, wird augenscheinlich, was bei der Auseinandersetzung über den Stellenwert beider Formen der Aufmerksamkeit auf dem Spiel zu stehen scheint. Aus dieser Perspektive heraus lässt sich die Durchsetzung der unwillkürlichen gegenüber der willkürlichen nur als Rückfall in eine primitive Stufe der Menschheitsentwicklung verstehen. Ohne diese Bewertung zu teilen, findet sich bei Dieter Claessens (1980: 64ff) der Hinweis, dass die im Laufe der Evolution nicht mehr länger einseitig auf Flucht ausgerichtete Aufmerksamkeit diese frei setzt, disponibler macht und damit erst die Hinwendung zu den eigenen Artgenossen ermöglicht. 


\section{Das Verlangen nach Sichtbarkeit in der visuellen Kultur}

Neben zahlreichen anderen Angeboten lässt sich unsere Gegenwartsgesellschaft treffend als „Kommunikationsgesellschaft“ (Münch 1991; Münch 1995; Serres 1991ff; Vattimo 1992) charakterisieren. Die visuelle Kultur tritt dabei nicht in Konkurrenz zur Kommunikationsgesellschaft, sondern ist Ausdruck derselben. Die Rede vom „,iconic“ (Boehm 1994; Maar / Burda 2004) oder ,pictorial turn“ (Mitchell 2008 a) meint dann, dass Kommunikation in zunehmendem Maße über visuelle Botschaften stattfindet. Wenngleich die Sprache keineswegs gänzlich verdrängt worden ist, spricht doch vieles dafür, dass das Visuelle kontinuierlich an Bedeutung zugenommen hat. Schon in den 1920er Jahren sieht der Filmtheoretiker Bela Balázs mit der Entwicklung der Kinematographie eine „visuelle Kultur“ (Balázs 2001: 104) entstehen. In den 50er Jahren bereits beklagt Günter Anders, dass wir ,einem Dauerregen von Bildern ausgesetzt sind" (Anders 1992: 250). In den 70er Jahren konstatiert der Kunsthistoriker John Berger, dass es „,in keiner anderen Gesellschaftsform der Geschichte [...] eine derartige Konzentration von Bildern [...], eine derartige Dichte visueller Botschaften“"(Berger 1974: 122) gegeben habe. In den 80er Jahren schließlich äußert der Historiker Christopher Lasch die Befürchtung, dass „,das Wuchern der visuellen und auditiven Bilder“ die Gesellschaft in eine „Gesellschaft der Show”“ (Lasch 1995: 80) verwandelt habe.

Wenn bereits im 20. Jahrhundert von einer nie da gewesenen Fülle an visuellen Botschaften die Rede ist - um wie viel mehr muss dies dann erst für unsere unmittelbare Gegenwart zutreffen, die nicht nur über mehr Technologien zur Bildproduktion verfügt als dies zwischen 1920 und 1980 der Fall gewesen ist, sondern diese auch in einem vor noch gar nicht so langer Zeit kaum für möglich gehaltenen Ausmaß privatisiert und unter die Leute gebracht hat? Neu an der gegenwärtigen Form einer visuellen Kultur dürfte sein, dass wir es nicht nur mit einer quantitativen Steigerung von immer mehr Bildern zu tun haben, wie dies bereits im 20. Jahrhundert beobachtet wurde, sondern auch mit einem qualitativen Umbruch durch einen Wandel in der Bildproduktion: Ein Großteil der Weltbevölkerung befindet sich nicht mehr nur in der Rolle des Zuschauers, der sich einer stetig steigenden Flut von Bildern ausgesetzt sieht, sondern auch in der Rolle des Produzenten und Senders visueller Botschaften. Damit wiederholt sich eine Entwicklung, die Walter Benjamin für die Schriftkultur wie folgt beschrieben hat:

,,Jahrhunderte lang lagen im Schrifttum die Dinge so, daß einer geringen Zahl von Schreibenden eine vieltausendfache Zahl von Lesenden gegenüberstand. Darin trat gegen Ende des vorigen Jahrhunderts ein Wandel ein [...], und es liegt heute so, daß es kaum einen im Arbeitsprozeß stehenden Europäer gibt, der nicht grundsätzlich irgendwo Gelegenheit zur Publikation einer Arbeitserfahrung, einer Beschwerde, einer Reportage oder dergleichen finden könnte. Damit ist die Unterscheidung zwischen Autor und Publikum im Begriff, ihren grundsätzlichen Charakter zu verlieren. [...] Der Lesende ist jederzeit bereit, ein Schreibender zu werden. " (Benjamin 1977: 155)

Analog zur Auflösung der Unterscheidung von Autor und Lesepublikum ist spätestens mit der Verbreitung der digitalen Fotografie und dem Aufkommen der Foto-Handys auch die Unterscheidung von Bildproduzenten und Bildkonsumenten gekippt. Mit anderen Worten: Der Fotografierte ist jederzeit bereit, ein Fotograf zu werden. Das Foto-Handy hat jeden einzelnen Besitzer eines solchen Geräts dazu in die Lage versetzt, Ereignisse, Personen und Gegenstände sichtbar zu machen, die sonst unsichtbar geblieben wären. Wenn die auf diese Weise angefertigten Dokumentationen über Internetportale wie etwa „YouTube“ verbreitet werden, erfahren sie eine Aufmerksamkeit, die ohne die oft verwackelten und verschwommenen Szenefetzen niemals zu erreichen gewesen wäre. Ein Großteil der Bilder, die wir heute über Tsunamis, Terroranschläge, Aufstände, Revolutionen und andere Ereignisse des Weltgeschehens zu sehen bekommen, stammen immer seltener von den Profireportern der Nachrichtenagenturen und immer häufiger von Passanten, Touristen und den Teilnehmern der Revolten und Protest- 
veranstaltungen selbst. Wir erleben damit die Entstehung eines neuen Bürgerjournalismus mit weit reichenden Folgen: Wer immer ein Ereignis gefilmt hat, das für die Öffentlichkeit von Interesse sein könnte, stellt die Bilder auf Internetseiten wie „iReport.com“ zur Verfügung oder lädt sie bei „YouTube“ hoch. Damit steigt insgesamt die Anzahl von Ereignissen, die visuell erfasst und fotografisch-filmisch festgehalten werden, um ein Vielfaches (vgl. Schroer 2012).

Vor dem Hintergrund dieser enormen Ausweitung der Dokumentation von Ereignissen verstärkt sich der für eine visuelle Kultur konstitutive Eindruck, dass nur das existiert, was auf Fotos und Filmen festgehalten wird: „Leben heißt fotografiert werden und Aufzeichnungen vom eigenen Leben zu besitzen“ heißt es bei Susan Sontag (SZ 24.5.2004) dazu treffend. Es ist diese Überzeugung, die zu einem wahren Ringen um Sichtbarkeit führt, da ein von Kameras nicht erfasstes Dasein die Exklusion aus der Medien- und Kommunikationsgesellschaft riskiert:

„, In einer Gesellschaft, in der alles durch Kommunikation bewegt wird, kann sich niemand mehr dem Zwang zur Erzielung von öffentlicher Aufmerksamkeit entziehen. Anderenfalls ist man vergessen und verloren. Wer sich nicht gut darstellen kann, hat in dieser Gesellschaft keine Chance. " (Münch 1991: 17)

Angesichts der grassierenden Angst davor, nicht wahrgenommen zu werden und unsichtbar zu bleiben, ist in der Literatur von „Darstellungszwänge[n]“ (Münch 1995: 124), „Visualisierungsdruck“ (Meyer / Kampmann 1998: 45), ,Sichtbarkeitsdruck“ (Holert 2000: 13) und einem „Trend gesteigerter Visibilitätszwänge“ (Reichert 2008: 7) die Rede. Damit scheint gemeint zu sein, dass die Visualisierung und Darstellung des Selbst dem Einzelnen von außen abverlangt wird, nicht aber seinen eigenen Interessen entspricht. Nimmt man dagegen den Begriffsvorschlag von Anthony Giddens (1992: 228ff) auf, der Zwang nicht nur als einschränkend, sondern auch als ermöglichend versteht, dann lässt sich damit die Gleichzeitigkeit eines gesellschaftlichen Imperativs und eines Bedürfnisses der Individuen nach Ausdruck und Erscheinen sehr viel plausibler charakterisieren. Denn was bei der Rede von Druck und Zwang übersehen wird ist, dass besagter Zwang auf einen tief verankerten ,,Drang zur Selbstdarstellung'" trifft, wie dies Hannah Arendt im Anschluss an Adolf Portmann (Arendt 1979: 39)5 genannt hat, ohne den das Leben gar nicht vorstellbar sei und - wie sich hinzufügen lässt ohne den der Zwang wohl auch kaum derart wirksam werden könnte. Es gehört demnach zu den Grundtatsachen der conditio humana, dass der Mensch sich nur über die Wahrnehmung der anderen selbst gewahr werden kann. Um diese Wahrnehmung zu ermöglichen, muss er sich bemerkbar, muss er auf sich aufmerksam machen: Der Mensch, so heißt es bei Helmuth Plessner ausdrücklich, ,muß spielen, etwas vorstellen, als irgendeiner auftreten, um die Aufmerksamkeit auf sich zu lenken und sich die Achtung der Anderen zu erzwingen“"(Plessner 2003: 82). Da ihm nichts so unerträglich ist wie das „Nichtbeachtetsein“(Plessner 2003: 64), ist er gezwungen, nach außen in Erscheinung zu treten, sich zu zeigen, sich darzustellen und zu inszenieren. Die pure Sichtbarkeit des Menschen, die Hans Blumenberg in seiner „Be-

5 „Was sehen kann, möchte gesehen werden; was hören kann, möchte gehört werden; was berühren kann, möchte sich berühren lassen. Es ist gerade so als hätte alles, was lebt [...], einen Drang, zu erscheinen, sich in die Welt der Erscheinungen einzufügen, indem es [...] sich als Individuum darstellt und zeigt“" (Arendt 1979: 39). Kurz zuvor heißt es: „Leben heißt von einem Drang zur Selbstdarstellung beherrscht sein, der Reaktion auf die eigene Erscheinungshaftigkeit“ (Arendt 1979: 31). Ähnlich notiert Blumenberg (2002: 172): „Man denke daran, dass sogar ein Land, eine Nation, ein Staat ein Bedarf ,zu erscheinen' haben." Der Kampf um Aufmerksamkeit lässt sich insofern auch als eine Reaktion auf schwindende „Erscheinungschancen“ interpretieren: „Der fundamentalpolitische Charakter der menschlichen Erscheinungschancen ist in jüngerer Zeit daran aufgewiesen worden, dass die modernen Aufmerksamkeits- und Auffälligkeitsregimes viele Menschen auf den Nullpunkt des Erscheinens also die Grenze zum Verschwinden - reduzieren." (Seitter 2001: 178). 
schreibung des Menschen“ (Blumenberg 2006: 777ff) als anthropologische Konstante gilt, garantiert ihm keineswegs schon die Aufmerksamkeit der Anderen. Denn nichts spricht dafür, dass das prinzipiell Sichtbare auch wirklich gesehen wird. Der Schritt von der bloß potenziellen Sichtbarkeit zum wirklichen Sichtbar-Sein erfordert vielmehr die gezielte Aufmerksamkeit durch ein nahes oder auch entferntes Gegenüber. Zumal in einer Kommunikations- und Mediengesellschaft, in der die Blicke nicht mehr allein zwischen menschlichen Akteuren ausgetauscht, sondern in verstärktem Maße auf Monitore gerichtet werden, face-to-face-Interaktionen durch face-to-screen-Interaktionen ergänzt und teilweise auch ersetzt werden (vgl. KnorrCetina / Brügger 2005), bedarf es zusätzlicher Anstrengungen, um in das Wahrnehmungsfeld menschlicher Akteure zu gelangen.

Die Anstrengungen, die unternommen werden, um sich gegen die Mitbewerber im Wahrnehmungsfeld des Gegenübers durchzusetzen, können auf den verschiedenen sozialen Feldern (vgl. Bourdieu 2001) beobachtet werden, auf denen mit unterschiedlichen Mitteln um Aufmerksamkeit gekämpft wird. ${ }^{6}$ Jedes soziale Feld, so meine Annahme, verfügt über ein ihm eigenes Medium zur Erlangung von Aufmerksamkeit bzw. Sichtbarkeit. In einem Feld sichtbar wird, wer es versteht, die Aufmerksamkeit des jeweiligen Publikums auf sich zu ziehen: Politiker erlangen Sichtbarkeit, indem sie bestimmte Vorschläge zu politischen Sachverhalten machen und damit mit Hilfe der Medien an die Öffentlichkeit treten; Wissenschaftler werden im wissenschaftlichen Feld sichtbar, wenn es ihnen gelingt, ihre Gedanken, Ideen, Einfälle und Entdeckungen in einschlägigen Fachzeitschriften publik zu machen. Je renommierter der Publikationsort ist, desto höher ist die Wahrscheinlichkeit, dass davon nicht nur das eingeweihte Fachpublikum, sondern auch die breitere Öffentlichkeit Kenntnis erhält. Die Massenmedien fungieren hier als Vermittler zwischen beiden Öffentlichkeiten, indem sie in Fachzeitschriften publizierte Forschungsergebnisse in kondensierter und vereinfachter Form referieren; Künstler erreichen Sichtbarkeit, indem sie mit spektakulären Performances und aufwendigen Inszenierungen die Aufmerksamkeit auf ihr Werk und sich selbst zu lenken versuchen; Religionsführer machen sich durch aufrüttelnde Predigten und pompöse Auftritte sichtbar; Sportler durch immer neue Höchstleistungen, Rekorde und Medaillen; Mediziner durch gewagte, bisher nicht vorgenommene Operationen; Richter durch in der Öffentlichkeit als zu lasch oder zu hart empfundene Urteile; Anwälte durch prominente Mandanten; Köche durch ausgefallene Rezepturen; Architekten durch spektakuläre Bauten usw. Zusammengenommen unterstreichen all diese Praktiken die fundamentale Einsicht „Esse est percipi“ (George Berkely), die bei Pierre Bourdieu mit folgenden Worten wieder aufgenommen wird: „Die soziale Welt ist auch Wille und Vorstellung, und sozial existieren heißt auch, wahrgenommen werden und als distinkt wahrgenommen werden.“ (Bourdieu 1990: 99) ${ }^{7}$ So unterschiedlich dabei die jeweiligen Medien auch sein mögen, mit denen um Aufmerksamkeit geworben wird, so übereinstimmend setzen die Akteure auf den Einsatz von Bildmaterial, um sich ins begehrte Licht der Öffentlichkeit zu rücken (TV-Auftritte, die eigene Homepage, Fotoberichte in Zeitschriften etc.). Allen Fällen ist außerdem gemeinsam, dass die Aus- und Durchführung der jeweiligen Handlungen allein nicht mehr länger ausreichen, wenn man in seinem Metier aus der Masse konkurrierender Angebote herausragen will. Notwendig sind vielmehr besondere Darstellungsanstrengungen und Inszenierungsbemühungen, die jede Handlung begleiten müssen, um in den Aufmerksamkeitsfokus des potenziellen Publikums zu

6 Der Prozess gesellschaftlicher Differenzierung, wie er schon von den Klassikern der Soziologie (Weber, Durkheim, Simmel) beschrieben worden ist, lässt sich als Antwort auf das Problem verstehen, dass nicht allen Prozessen und Ereignissen gleichzeitig und von einer dominierenden Instanz aus Aufmerksamkeit geschenkt werden kann. Die Ausdifferenzierung unterschiedlicher Wertsphären, sozialer Systeme oder Felder geht mit der Herausbildung von Sonderaufmerksamkeiten einher, die für den jeweiligen Sektor exklusiv gelten.

7 Mit direktem Bezug zu Berkeley, der als Erfinder des Immaterialismus gilt, vgl. Bourdieu (1998: 16). Zum Prinzip des „esse est percipi“ vgl. auch Türcke (2002: 38ff). 
gelangen. Handlungen verlieren insofern ihren reinen Aktionscharakter und werden überformt von expliziten Inszenierungsanstrengungen (vgl. Soeffner 2004, Willems 1998). Im Zeitalter der Massenmedien erfahren solche bereits auf der Ebene von face-to-face-Interaktion zu beobachtende Inszenierungsanstrengungen (vgl. Goffman 2009; Hitzler 1992, 1998) eine zusätzliche Steigerung, da diese vor dem Hintergrund globaler Konkurrenzverhältnisse nur mehr das wahrnehmen, aufgreifen und senden, was jenseits des Gewöhnlichen, Normalen und Immergleichen liegt. Deshalb werden Handlungen bereits auf die öffentliche Aufmerksamkeit hin inszeniert und entsprechend medienkompatibel aufbereitet (vgl. Lasch 1995: 80; Welsch 1990: 58). Durch diese zunehmende Veröffentlichungspraxis geraten immer mehr Aktivitäten in den Fokus der öffentlichen Aufmerksamkeit. Eine Folge dieser sukzessiven Erweiterung der öffentlichen Sphäre ist eine enorme Ausweitung der Starzone (vgl. Schroer 2010: 389). Stars kennen wir nicht mehr nur aus dem traditionellen Unterhaltungs- und Showgeschäft. Vielmehr kommt es längst quer durch alle sozialen Felder zum Aufbau von Stars, sodass wir es mittlerweile auch mit Polit-Stars, Stararchitekten, Staranwälten und Starköchen zu tun haben. Die enorme Steigerung von Angeboten, denen Aufmerksamkeit entgegengebracht werden soll, kann von den begrenzten Aufmerksamkeitskapazitäten der Akteure jedoch immer weniger eingeholt werden. Was wir somit erleben, ist eine Neuauflage des bereits von Georg Simmel konstatierten unausgeglichenen Verhältnisses von subjektiver und objektiver Kultur, das die Notwendigkeit zur selektiven Aufmerksamkeit gerade auf den Plan ruft. ${ }^{8}$ Als Antwort auf diese systematische Überforderung der Aufmerksamkeitsfähigkeit lässt sich die Verbreitung von Aufmerksamkeitsspeichern verstehen, die in immer mehr private Hände gelangen. Allgegenwärtige Fotohandys und Kleinstkameras machen es möglich, immer mehr Situationen, Ereignissen, Personen und Dingen vorübergehend Aufmerksamkeit entgegen zu bringen, indem man sie fotografisch und filmisch mit der Absicht festhält, ihnen zu einem späteren Zeitpunkt die volle Aufmerksamkeit angedeihen zu lassen. Obwohl es dazu in vielen oder sogar den meisten Fällen womöglich gar nicht kommt, ist für unseren Zusammenhang allein das Vorhaben entscheidend, denn es vermag die Überforderungen der Fähigkeit zur Aufmerksamkeit des Einzelnen und seine Suche nach Entlastung durch die Verwendung von Aufmerksamkeitsmaschinen ${ }^{9} \mathrm{zu}$ unterstreichen.

\section{Aufmerksamkeit als knappe Ressource - Werbung für das eigene Ich}

Was in den Betrachtungen Hannah Arendts und Helmuth Plessners noch als nahezu selbstverständlich unterstellt, jedenfalls nicht weiter problematisiert wird, ist das Vorhandensein eines Publikums, das den jeweiligen Darbietungen Aufmerksamkeit schenkt. Dazu noch einmal Hannah Arendt: „Es gibt in dieser Welt nichts und niemanden, dessen bloßes Sein nicht einen Zuschauer voraussetzte. Mit anderen Worten, kein Seiendes, sofern es erscheint, existiert für sich allein; jedes Seiende soll von jemanden wahrgenommen werden." (Arendt 1998: 29) Schon für Interaktionsbeziehungen unter Anwesenden gilt, dass die Aufmerksamkeit der anderen nicht selbstverständlich zur Verfügung steht. Anwesenheit allein genügt nicht, um die

8 In seinem Aufsatz „Der Begriff und die Tragödie der Kultur“ heißt es: „Der ins Unabsehbare wachsende Vorrat des objektivierenden Geistes stellt Ansprüche an das Subjekt, weckt Velleitäten in ihm, schlägt es mit Gefühlen von eigener Unzulänglichkeit und Hilflosigkeit, spinnt es in Gesamtverhältnisse, deren Ganzheit es sich nicht entziehen kann, ohne doch ihre Einzelinhalte bewältigen zu können. So entsteht die typische problematische Lage des modernen Menschen: das Gefühl, von einer Unzahl von Kulturelementen umgeben zu sein, die für ihn nicht bedeutungslos sind, aber im tiefsten Grunde auch nicht bedeutungsvoll; die als Masse etwas Erdrückendes haben, weil er nicht alles einzelne innerlich assimilieren, es aber auch nicht einfach ablehnen kann, da es sozusagen potentiell in die Sphäre seiner kulturellen Entwicklung gehört.“ (Simmel 1996: 412).

9 Aufmerksamkeitsmaschinen (Überwachungskameras, Bewegungsmelder etc.) übernehmen die Rolle des stets aufmerksamen Beobachters und ersetzen damit in Teilbereichen die notorisch knappe, schnell erlahmende Aufmerksamkeitsfähigkeit des Menschen. 
geschätzte Aufmerksamkeit der anderen auf sich zu lenken: „Der Mensch kann Andere in ihrer Anwesenheit unmittelbar erfahren, muß es aber nicht. Wenn er jemandem begegnet, kann er es zwar nicht vermeiden, ihn unmittelbar zu erleben, er braucht sich aber diesem Erlebnis nicht unbedingt zuzuwenden [...]. Wenn A nichts mit B zu tun hat und mit ihm nichts zu tun haben will, kann er auch in seiner Anwesenheit innerlich - unter Umständen sogar äußerlich - die Augen schließen und an gar nichts oder sein Mittagessen oder auch an C denken. Und umgekehrt" (Schütz / Luckmann 2003: 555). Obwohl wir uns also der Wahrnehmung des Anderen durch seine unmittelbare Anwesenheit kaum entziehen können, muss daraus noch lange keine bewusste Hinwendung resultieren. Aktuell ist diesbezüglich eine Steigerung der Möglichkeiten zu beobachten, sich in face-to-face-Situationen mit Hilfe zahlreicher technischer Artefakte (Laptop, Handy und MP3-Player mit Kopfhörer usw.) vor der unerwünschten Hinwendung der anderen zu schützen und sich als quasi nicht anwesend zu präsentieren. Angesichts des derart unverlässlich gewordenen Publikums für die alltäglichen Aufführungspraktiken der nach Ausdruck suchenden Darsteller, sucht sich der „Drang nach Selbstdarstellung“ neue Wege - und findet sie in den Medien, die nicht nur eine Selbstdarstellung vor möglichst vielen Zuschauern ermöglichen, sondern auch deren Reaktionen dokumentieren und zu einer gewissen Verstetigung der Selbstdarstellungsbemühungen und ihrer Reproduktion führen. ${ }^{10}$ Dagegen erscheint die Aufmerksamkeit in Interaktionsbeziehungen unter Anwesenden im face-toface-Kontakt doch eher flüchtig und labil zu sein. Allerdings muss die mediale Selbstdarstellung, will sie sozial wirksam werden, durch das enge Nadelöhr der (öffentlichen) Aufmerksamkeit, die Zygmunt Bauman als „,knappste Ressource in der politischen Ökonomie der Postmoderne" (1995: 20) gilt. Es ist diese vielfach angeführte Knappheit der Aufmerksamkeit, die dafür sorgt, dass wir es mit immer pointierter ausfallenden Selbstdarstellungen zu tun bekommen, ja eine Spirale sich permanent überbietender Selbstinszenierungen beobachten können, die allesamt das Ziel verfolgen, nicht ignoriert werden zu können. Im Kultfilm „Seven“ von David Fincher erläutert der von Kevin Spacey verkörperte Serienkiller gegen Ende des Films: „Wenn man heute Menschen dazu bewegen will, einem zuzuhören, reicht es nicht mehr aus ihnen sachte auf die Schulter zu klopfen. Man muß auf sie mit einem Vorschlaghammer einschlagen." Diese Einsicht war allerdings schon dem Nationalökonomen Werner Sombart bekannt: Der Grundsatz aller modernen Geschäftsführung sei „1. die Aufmerksamkeit, 2. die Kauflust der Kunden zu erregen. Das erste geschieht dadurch, dass man ihnen möglichst laut in die Ohren schreit oder mit möglichst grellen Farben in die Augen sticht" (Sombart 1988: 178). Die beiden auf den ersten Blick ähnlich klingenden Einsichten sind jedoch im ersten Fall von der Produzenten- und im zweiten Fall von der Rezipientenseite aus formuliert. Während sich hinter der ersten Aussage die Erfahrung verbirgt, dass die Aufmerksamkeit der Menschen allenfalls noch mit äußerst drastischen Mitteln geweckt werden kann, wird in der zweiten Aussage die Zumutung betont, die in der Anwendung solch aggressiver Werbemaßnahmen für den Rezipienten bzw. Konsumenten liegt. Neu für unseren gegenwärtigen „Kulturkapitalismus“ (Rifkin 2000; Žižek 2002) dürfte sein, dass die für den Verkauf von Waren ersonnenen Werbemaßnahmen längst auch auf menschliche Akteure Anwendung finden, wie Zygmunt Bauman betont: „Hinter dem Traum vom Berühmtsein verbirgt sich ein anderer Traum, der Traum, [...] sich in eine beachtenswerte, beachtete und begehrte Ware zu verwandeln, eine Ware, über die man spricht und die sich von der Masse der Waren abhebt, eine Ware, die man unmöglich übersehen, verlachen, entlassen kann" (Bauman 2009: 22). Wer darin nur eine Verdinglichung des Menschen zur Ware zu erkennen vermag, übersieht, dass wir umgekehrt eine Verwandlung von Konsumobjekten in Persönlichkeiten beobachten können (Bolz 2002: 108), was unterstreicht, wie sehr sich Ding und Mensch im Konsumkapitalismus aufeinander zubewegen. Waren wollen eben nicht nur verkauft und erworben, sondern auch umworben,

10 So kann etwa das visuelle Material nicht nur beliebig oft angesehen, sondern auch mit Bewertungen und Kommentaren versehen werden. 
gepflegt und bewundert werden. Warum, ließe sich deshalb fragen, sollte nicht jeder Einzelne aus sich und seinem Leben eine Ware machen dürfen? Und das bedeutet in einer visuellen Kultur vor allem, aus sich als Bild eine Ware zu machen. Im Internet haben sich längst Formate etabliert, die es jedem User ermöglichen, für sein eigenes Ich zu werben und um die knappe Aufmerksamkeit der anderen Netzteilnehmer zu buhlen. Portale wie „Facebook“, „YouTube“, „MySpace“, „StudiVZ“ usw. sind wahre Bühnen der Selbstdarstellung und Selbstinszenierung (vgl. Neumann-Braun 2011). Will man gegen die starke Konkurrenz überhaupt eine Chance haben, an der knappen Ressource Aufmerksamkeit zu partizipieren, die als neue Währung des Internets gehandelt wird, muss man sich immer wieder etwas Neues einfallen lassen - gilt doch gerade das Überraschende, allen Erwartungen und Routinen Zuwiderlaufende nahezu als Garant für die Erzielung von Aufmerksamkeit, während die Gewohnheit als ihr ältester Feind gehandelt wird. ${ }^{11}$ Internetplattformen wie „My Space“, „YouTube“ u.a. - aber auch TV-Formate wie „Big Brother“, „Deutschland sucht den Superstar“ oder „Ich bin ein Star - Holt mich hier raus!" - zeigen in beeindruckender Weise das Ausmaß der Schau- und Zeigelust, von dem die Individuen in der medialen Kommunikationsgesellschaft erfasst worden sind (vgl. Schroer 2003; Hitzler 2007). Da in einer „visuellen Kultur“ nur zählt, wer sichtbar ist, wer angesehen wird, wer die Blicke der Anderen immer wieder auf sich zu ziehen vermag, gibt es das schier unermüdliche Bestreben, in den Fokus einer Kamera zu gelangen, denn fotografiert oder gefilmt zu werden, heißt Bedeutung zu erlangen (vgl. Baudrillard 1992: 60ff).

Angesichts der scheinbar unbegrenzten Offenheit, mit der auf den Netzseiten auch intime Details aus dem eigenen Leben präsentiert werden, ist von einem „Sieg des Exhibitionismus“ (ZEIT 27.4.2000), der „Tyrannei der Intimität“ (Sennett 1983) oder auch von der ,schamlosen Gesellschaft“" (vgl. Spiegel 2 / 1993; Ernst 1997) die Rede. Dass es überhaupt (zumeist junge) Menschen gibt, die sich an diesem Spiel beteiligen, wird mit einer Mischung aus Unverständnis, Bedauern und Häme kommentiert. Der Vorwurf lautet dabei vor allem, dass diese Klientel von Usern keinen Sinn für das Private mehr habe und diese Errungenschaft des bürgerlichen Zeitalters achtlos über Bord werfe, wenn es sich derart ungehemmt vor der Öffentlichkeit entblöße.

Die Idee des Individuums lebt nun in der Tat davon, dass ein Teil des Ichs stets intransparent für die anderen bleiben muss, will man den Anspruch auf Individualität nicht aufgeben. Als Regel kann mit Georg Simmel gelten: „Was nicht verborgen wird, darf gewußt werden, und: was nicht offenbart wird, darf auch nicht gewußt werden“ (Simmel 1992: 396). Simmel geht davon aus, dass innerhalb des modernen Lebensstils der einzelne geschützt ist vor dem ,gegenseitigen Hineinsehen und Sichhineinmischen“ (Simmel 1992: 412), wie es für unentwickeltere Zustände, die sich im dörflichen Zusammenleben noch gehalten haben, nach wie vor der Fall ist. Zivilisiertes und urbanes Zusammenleben gelingt für ihn erst auf der Basis des gleichzeitigen „Sich-Offenbarens und Sich-Zurückhaltens“ (Simmel 1992: 402), eines diffizilen Gleichgewichts zwischen „Verbergen“ und „Enthüllen“ (Simmel 1992: 410).

11 Trotz ihrer vielfach unterstellten Gegnerschaft verweist Walter Benjamin auf die Notwendigkeit der Transformation von Aufmerksamkeit in Gewohnheit und umgekehrt: „Die erste aller Eigenschaften, sagt Goethe, ist die Aufmerksamkeit. Sie teilt jedoch den Vorrang mit der Gewohnheit, die ihr vom ersten Tage an das Feld bestreitet. Alle Aufmerksamkeit muß in Gewohnheit münden, wenn sie den Menschen nicht sprengen, alle Gewohnheit von Aufmerksamkeit verstört werden, wenn sie den Menschen nicht lähmen soll.“ (Benjamin 1972: 407) Einen ähnlichen Zusammenhang stellt William Isaac Thomas her: ,Wenn die Gewohnheiten reibungslos laufen, so lässt die Aufmerksamkeit nach; sie arbeitet nicht mehr. Wenn aber etwas passiert, das den Gang der Gewohnheit unterbricht, so wird die Aufmerksamkeit auf den Plan gerufen und bringt eine neue Verhaltensform hervor, durch welche die Krise überwunden wird. Die Funktion der Aufmerksamkeit besteht also darin, neue und angemessene Gewohnheiten zu schaffen.“(Thomas 1965: 285). 
Wenn man sich die medialen Selbstenthüllungsformate noch einmal vergegenwärtigt, so mag es zunächst den Anschein haben, als hätte sich das von Georg Simmel betonte Zusammenspiel von Enthüllen und Verbergen zur Seite der Enthüllung hin aufgelöst (vgl. Jung / Müller-Doohm 1998). Dem ausgestellten Individuum scheint die von Simmel zum Individualitätsgaranten erhobene Kategorie des Geheimnisses nichts mehr zu sagen. Auf einen privaten Schutzraum verzichtet es leichtfertig, so der immer wieder zu hörende Vorwurf.

Doch bei genauerem Hinsehen fällt auf, dass selbst in den scheinbar gänzlich hemmungslosen Enthüllungen immer noch ein Quäntchen Verborgenes enthalten ist. Manch eine Webcam, die angeblich zum Zwecke einer lückenlosen Überwachung der Privaträume eines Individuums installiert wurde, macht vor einigen Orten der Wohnung durchaus Halt und der freiwillig Beobachtete behält sich die Kontrolle über das An- und Abschalten der Kameras vor, wechselt zwischen online-Sein und offline-Sein (vgl. Rössler 2001: 279); die Bewohner der ersten Staffel des Endemolschen Big Brother Containers in Köln Hürth haben sich entgegen des Versprechens völliger Transparenz mit Hilfe aufgehängter Handtücher ein winziges Fleckchen Intimität zu retten versucht und die Computerbranche lebt nicht nur vom Verkauf von Überwachungstechnologie, sondern auch vom Verkauf von Software, mit deren Hilfe ein anonymes Surfen im Netz ermöglicht werden soll. Zusammengenommen unterstützen diese Beispiele die folgende Einsicht des französischen Philosophen und Wissenschaftstheoretikers Michel Serres: „Selbst die Ärmsten der Armen bewahren sich ein Mindestmaß an Privatem, das es ihnen ermöglicht, wenigstens einige Augenblicke für sich zu sein. Ob Lumpen oder Nische, das ist das Residuum an Eigenem, das proprium, das die Menschen definiert: ein Mindestmaß, ein Rest, eine Spur, ein Residuum, die einzige differentia specifica. Ein vollkommen öffentliches Leben würde uns zerstören, totale Öffentlichkeit wäre tödlich." (Serres 2005: 50) Aus den hier nur angedeuteten Möglichkeiten zur Wahrung der Privat- und Intimsphäre durch die gewollte Herstellung von Unsichtbarkeit ergeben sich eine Reihe von Fragen: Ist es denkbar, dass selbst hinter den scheinbar radikal exhibitionistischen Auftritten der Versuch steckt, sich gleichzeitig ,,maximal sichtbar zu machen und zu verhüllen“ (Plessner 2003: 85)? Stellen die Selbstinszenierungen vielleicht den Versuch dar, einerseits den an ein jedes Individuum gestellten Visualisierungsanforderungen gerecht zu werden und andererseits dennoch einen Teil seines Selbst für sich zu bewahren? Soll vielleicht gerade durch die extrovertierte Zurschaustellung privater und intimer Informationen von dem abgelenkt werden, was man wirklich für privat und intim hält? Wird als privat einfach längst nicht mehr das angesehen, was die Verteidiger der Privatheit als solches ausgeben? Suchen wir das Private womöglich an der falschen Stelle? Verstellt uns die tradierte Entgegensetzung von ,privat" und „öffentlich“ womöglich den Blick auf die Bedeutungsverschiebungen, die diese Kategorien gegenwärtig erfahren? Momentan überwiegt die Diagnose einer exzessiven Enthüllungsbereitschaft und der Lust an der Preisgabe der intimsten Details aus dem Alltag der Individuen. Den Sieg der Transparenz und der Sichtbarkeit zu verkünden und das Ende der Privatheit und des Geheimnisses auszurufen, wie es im Feuilleton und den dramatisierenden Darstellungen auch soziologischer Beobachter geschieht (vgl. Sofsky 2007), erfolgt jedoch vorschnell. Statt sich den immer gleichen, nahezu reflexhaft vorgebrachten Verlustanzeigen und Verabschiedungsritualen hinzugeben, halte ich es für sehr viel lohnender, sich mit der Neujustierung der Begriffe zu beschäftigen, die aufgrund der veränderten Praktiken notwendig erscheinen; mit der Frage also etwa, was die Unterscheidung Privatheit / Öffentlichkeit im 21. Jahrhundert bedeutet und mit welchen Konnotationen sie von den Akteuren belegt wird. An Stelle der verkrampften Verteidigung des Althergebrachten hätte dabei jene Neugier zu treten, die in den Selbstverlautbarungen der Soziologie oftmals als eine ihre ureigensten Aufgaben angesehen wird (vgl. Berger 1977: 27ff). 


\section{Sichtbar / Unsichtbar - Ein Analysemodell zur Aufmerksamkeit in der visuellen Kultur}

Die vorangegangenen Überlegungen sollten deutlich machen, dass die Rede von der visuellen Kultur keinen Sieg des Sichtbaren über das Unsichtbare mit sich bringt. Auch von einer völligen Preisgabe des Privaten und Intimen kann entgegen anders lautender Bekundungen der Zeitkritik keine Rede sein. Die als Exhibitionismus, Narzissmus und Schamlosigkeit deklarierten Verhaltensweisen lassen sich vor allem mit Hilfe der philosophischen Anthropologie als Darstellungsaktivität und Ausdruckssuche denken, die nunmehr vor dem Hintergrund und unter den Bedingungen einer visuellen Kultur stattfinden und mit den zur Verfügung stehenden Technologien in Gang gesetzt werden. Dass der Verweis auf psycho-pathologische Dispositionen zu kurz greift, wird schon allein daran erkennbar, dass der mit allen Mitteln der Darstellung geführte Kampf um Aufmerksamkeit nicht mehr länger nur auf menschliche Akteure bezogen bleibt, sondern längst auch auf Organisationen, Unternehmen, Wirtschaftsverbände, Gewerkschaften, Kirchen, Parteien, soziale Bewegungen, Vereine, Universitäten, Regionen, Länder, Staaten und Städte übergegriffen hat (vgl. Münch 1995: 114). Alle genannten Akteure befinden sich in einem wahren Überbietungswettbewerb um die knappe Ressource Aufmerksamkeit. Wem es dabei nicht gelingt, zumindest ein Mindestmaß an Sichtbarkeit für sich zu erlangen, der ist in einer Kommunikations- und Mediengesellschaft schlicht nicht existent. Typisch für eine Kommunikationsgesellschaft und eine visuelle Kultur ist insofern, dass Reden und Sichtbarkeit positiv, Schweigen und Unsichtbarkeit dagegen negativ konnotiert werden. ${ }^{12}$ Das heißt in beiden Fällen allerdings nicht, dass die jeweils negative Seite einfach verschwinden würde. Aus der Perspektive einer Theorie der Aufmerksamkeit muss hier vielmehr von Visualisierungsstrategien gesprochen werden, die stets auch neue Felder der Unsichtbarkeit erzeugen. Dabei gilt es an die Einsicht von Gilles Deleuze und Michel Foucault zu erinnern, das jedes Zeitalter Zonen der Sichtbarkeit und Zonen der Unsichtbarkeit hervorbringt (vgl. Deleuze 1992: 69ff). Gerade vor dem Hintergrund der Entstehung eines neuen Sichtbarkeitsregimes muss deshalb die Frage gestellt werden, wer und was unsichtbar bleibt und warum, denn Sichtbarkeit „,verdankt sich gesellschaftlichen und epistemologischen Möglichkeitsbedingungen, mit anderen Worten: Verhältnissen von Macht und Wissen“ (Holert 2000: 20). Um also nicht Sichtbarkeit gegen Unsichtbarkeit einfach auszuspielen, sollen sich mit Hilfe des folgenden Modells vier Möglichkeiten von Sichtbarkeit bzw. Unsichtbarkeit idealtypisch aufzeigen und analytisch unterscheiden lassen:

\section{Tabelle 1}

\begin{tabular}{|c|c|c|}
\hline & Sichtbar & Unsichtbar \\
\hline \multirow[t]{2}{*}{ Aktiv } & $\begin{array}{l}\text { 1. Präsenz (Beachtung) } \\
\text { Aufmerksamkeit erlangen } \\
\text { (Selbstdarstellung, Enthüllen) } \\
\text { (Bühne, Podest, Rahmen) } \\
\text { - INKLUSION - }\end{array}$ & $\begin{array}{l}\text { 2. Absenz } \\
\text { Aufmerksamkeit entfliehen } \\
\text { (Rückzug, Verbergen) } \\
\text { (Rückzugsräume) } \\
\text { - SELBSTEXKLUSION - }\end{array}$ \\
\hline & „Sichtbarkeitsfalle“ & \begin{tabular}{l|l} 
& „Unsichtbarkeitsfalle“
\end{tabular} \\
\hline Passiv & $\begin{array}{l}\text { 3. Überwachung } \\
\text { Ungewollt Aufmerksamkeit erlangen } \\
\text { (Panoptikon, Gefängnis) } \\
\text { - ZWANGSINKLUSION - }\end{array}$ & $\begin{array}{l}\text { 4. Irrelevanz (Missachtung) } \\
\text { Ungewollt keine Aufmerksamkeit erlangen } \\
\text { (Favelas, Slums, Banlieus) } \\
\text { - EXKLUSION - }\end{array}$ \\
\hline
\end{tabular}

Quelle: eigene Zusammenstellung

12 Für das Begriffspaar Reden und Schweigen vgl. Luhmann / Fuchs (1989). 
Die Aussage der vier Felder kann in folgenden Merksätzen zusammengefasst werden:

1. Wer / Was sich zu präsentieren versteht, kann die Aufmerksamkeit der anderen auf sich ziehen.

2. Wer / Was sich zu verbergen weiß, erfährt Entlastung von der Aufmerksamkeit der anderen.

3. Wer / Was dauerhaft Aufmerksamkeit auf sich zieht, wird überwacht.

4. Wer / Was keinerlei Aufmerksamkeit erfährt, gilt als irrelevant.

\section{Zur Erläuterung:}

\section{Feld 1) Präsenz: Sichtbar durch Selbstdarstellung, der von anderen Aufmerksamkeit gewährt wird}

Es gibt eine Fülle von Möglichkeiten, auf sich aufmerksam zu machen. Je nach Situation kann ein Pfeifen, können Schreie, laute Motoren und Handyklingeltöne dabei helfen, die Aufmerksamkeit auf sich zu lenken. Grundsätzlich verspricht alles dasjenige Aufmerksamkeit auf sich zu ziehen, was der Routine, dem Üblichen und Erwartbaren widerspricht. Auffälliges, Neues und Überraschendes hat gute Chancen, nicht ignoriert zu werden. ${ }^{13}$ Räumliche Arrangements wie etwa Rahmen für Bilder, Podeste für Vortragende oder Bühnen für Schauspieler tragen erheblich dazu bei, Aufmerksamkeit zu erzielen, weil sie die Blicke in die gewünschte Richtung lenken. Gelingt die Erzielung von Aufmerksamkeit, kann dies als gelungene Inklusion in die visuelle Kultur verstanden werden. Wie elementar das Erlangen von Aufmerksamkeit in einer mediensaturierten Gesellschaft ist, kann schon daran abgelesen werden, welch enormer Aufwand betrieben wird, um gesellschaftliche Sichtbarkeit zu erlangen. Die enorme Anzahl von Privatleuten betriebenen Homepages, das Bemühen von Unternehmen aller Art um einen gelungenen „Netzauftritt“, die Selbstdarstellungsbemühungen von Universitäten u.v.m. erklärt sich nur aus der existenziellen Notwendigkeit heraus, sich zu präsentieren. Längst hat sich die Ratgeberliteratur mit Titeln wie „Nie wieder unsichtbar“ (Säger 2009) oder „So machen Sie auf sich aufmerksam“ (Kirschner 1999) dem Thema angenommen.

Da jedoch nicht nur Aufmerksamkeit aufwenden anstrengend ist, sondern auch Aufmerksamkeit zu erhalten, entsteht aus der Situation, im Mittelpunkt der Aufmerksamkeit zu stehen, regelmäßig der Bedarf an Rückzugsmöglichkeiten. Damit gelangen wir von Feld 1 zu Feld 2:

\section{Feld 2) Absenz: Unsichtbar durch Rückzug von der Aufmerksamkeit der anderen}

$\mathrm{Zu}$ diesem Feld gehört das gesuchte Sich-verbergen, -verhüllen und -verstecken, das auch in einer vom Kampf um Aufmerksamkeit bestimmten Gesellschaft keineswegs zum Erliegen kommt: „Sich verbergen zu können, als anthropologisches Phänomen auf der Kehrseite der Visibilität, kann als Ansatz wenigstens zu dem Wunsch oder der Phantasie begriffen werden, nicht nur zeitweise aus dem Sichtfeld der anderen zu verschwinden, sondern die Last der verfolgbaren Identität überhaupt abzuwerfen“ (Blumenberg 2006: 804). Die Last der verfolgbaren Identität abzuwerfen findet sich als Sehnsucht manches Autors, der die Identifizierung von Werken insofern als Last empfindet, weil sie den Spielraum möglicher einzunehmender Standpunkte aufgrund einer geltenden Konsistenzregel einschränkt. In diesem Sinne fordert Foucault: „Man frage mich nicht, wer ich bin, und man sage mir nicht, ich solle der gleiche bleiben: das ist eine Moral des Personenstandes; sie beherrscht unsere Papiere. Sie soll uns frei

13 Neben den bereits genannten Autoren findet sich diese Einsicht bereits bei Immanuel Kant: „Durch das Neue, wozu auch das Seltene und das verborgen Gehaltene gehört, wird die Aufmerksamkeit belebt. [...] Das Alltägige oder Gewohnte löscht sie aus“" (Kant 2000: 55). 
lassen, wenn es sich darum handelt, zu schreiben." (Foucault 1973: 30) Der Wunsch nicht identifizierbar zu sein, kann aber auch bei Kriminellen angetroffen werden, die für ihre Taten nicht verantwortlich gemacht werden wollen. Mafiosi scheuen generell die Öffentlichkeit und ihre Anführer machen sich so unsichtbar wie nur möglich, durch Maskerade, Gesichtsoperationen und vollständiges Abtauchen. Darüber hinaus findet man in der Populärkultur und Kunstszene Beispiele für medienscheue Akteure, die sich der allgemeinen Forderung nach Sichtbarkeit (erfolgreich) entziehen. Der Regisseur Terence Malick, die Schriftsteller Patrick Süskind, Jerome Salinger oder Thomas Pynchon und die Pop-Gruppe „The Residents“ haben diesen Weg gewählt. Sich als Kulturschaffender dem Sichtbarkeitsgebot zu entziehen dürfte allerdings weniger schwer sein, als in der Rolle des Außenseiters Sichtbarkeit zu erlangen. Die gewählte Flucht vor der Sichtbarkeit setzt jedenfalls ein Bewusstsein dafür voraus, sichtbar zu sein: „Ein Verhalten, welches die Absicht sich zu verbergen zum Ausdruck bringt, impliziert Bewußtsein der Visibilität: erst wer weiß, dass er gesehen werden kann, kann es darauf anlegen, nicht gesehen zu werden.“(Blumenberg 2006: 803) Im Alltag dürfte es nach wie vor für jeden Situationen geben, in denen er oder sie sich wünscht, sich unsichtbar machen zu können. Schüler, die ihre Hausaufgaben nicht erledigt haben, versuchen dem Blick des Lehrers auszuweichen, um dessen Aufmerksamkeit nicht auf sich zu lenken und aufgerufen zu werden. Kinder, deren Eltern streiten, versuchen sich so unauffällig wie möglich zu benehmen, um nicht selbst zum Gegenstand des Streits zu werden. Hinzu kommen Situation der Scham, in der man sprichwörtlich, in den Boden versinken' möchte, um den Blicken der anderen nicht länger ausgesetzt zu sein. Kinder schließen einfach die Augen, wenn sie wünschen, nicht gesehen zu werden. Das berühmte, Abtauchen in der Menge' gehört ebenso in diesen Zusammenhang wie die Suche nach dem Schutz vor den neugierigen Blicken der anderen mit Hilfe von Masken, Schleiern, Sonnenbrillen und Rückzugsräumen. Diese Schutzartefakte bergen jedoch die Gefahr, die Aufmerksamkeit der Anderen erst recht zu provozieren. Wird die gesuchte Unsichtbarkeit übertrieben, der Rückzug zu radikal, sorgen Videoüberwachung und Datenkontrolle dafür, dass die Unsichtbarkeit nicht von Dauer ist, Informationen eingeholt und Transparenz hergestellt wird. Dann kann die vorübergehende Selbstexklusion aus den Belastungen des Sichtbarkeitsregimes in Zwangsinklusion in die visuelle Kultur umschlagen. Dies beschreibt den Weg von Feld 2 zu Feld 3:

\section{Feld 3) Überwachung: Sichtbarkeit durch die ungewollte Aufmerksamkeit der anderen}

Unfreiwillig und ununterbrochen den Blicken ausgeliefert zu sein, auf Schritt und Tritt kontrolliert zu werden, ohne Möglichkeiten des Rückzugs, zeichnet das dritte Feld aus, das von Bildern einer totalen Überwachungsgesellschaft lebt und durch George Orwells Roman „1984“ ebenso repräsentiert wird wie durch Jeremy Benthams Modell des „Panopticons“ (vgl. Foucault 1977), dessen Insassen sich der permanenten Sichtbarkeit ausgesetzt sehen. Nach beiden Modellen soll die Überwachung zum Wohlverhalten und zur Disziplinierung der Überwachten beitragen. Mit der Überwachung ist dabei immer eine gezielte Verhaltensänderung intendiert, die sich einstellen soll, weil man weiß, dass man beobachtet wird oder zumindest jeder Zeit beobachtet werden könnte. Doch woran in Orwells Modell in erster Linie gedacht wurde, war die Überwachung des Bürgers durch einen allmächtigen Staat. Und in der Tat hat dieser über seine gesamte Geschichte hinweg Informationen über seine Untertanen gesammelt und er hat bis heute in diesem Bestreben nicht nachgelassen (vgl. Giddens 1985). Längst stehen ihm dabei Mittel zur Verfügung, von denen Orwell noch gar nichts ahnen konnte. Vor allem die Möglichkeit der Vernetzung der aus unterschiedlichen Quellen bezogenen Daten stellt dabei einen entscheidenden Schritt in Richtung des ,gläsernen Bürgers“ dar. Aber inzwischen hat der Staat in seinen Überwachungsbestrebungen und -möglichkeiten Konkurrenz bekommen, haben pri- 
vate Überwachungen an Bedeutung gewonnen und ist ein florierender Markt rund um die Sicherheitstechnologien entstanden. Der Bürger als Konsument steht dabei eher im Mittelpunkt als der politische Bürger, der sich womöglich mit subversiven Umsturzgedanken trägt. Ob beim Gang in Banken, Casinos, Büros, Supermärkte, Gerichte, die nächste U-Bahn-Station oder beim Tanken: die Kamera ist omnipräsent. Doch ob es nun um das Ausspionieren politischer Verbindungen geht oder um die Durchleuchtung des Konsumenten zwecks gezielterer Kaufofferten: Wir sind umgeben von Technologien, die täglich Informationen über uns sammeln und ein Profil über unsere Interessen und Leidenschaften herzustellen in der Lage sind. Dem großen Bruder haben sich längst ,eine Myriade wohlwollender ,kleiner Schwestern "“ hinzugesellt (vgl. Castells 2002: 320) und aus dem Panopticon ist längst ein Synopticon (vgl. Mathiesen 1997) geworden, in dem nicht mehr wenige die vielen beobachten, sondern viele die wenigen. Während die klassischen Überwachungsszenarien, die noch von einer zentralen Instanz ausgingen, zunehmend von Positionen abgelöst wurden, die eine größer werdende Anzahl von Überwachungsinstanzen konstatierten, nähern wir uns inzwischen einem Zustand, in dem gewissermaßen jeder jeden beobachtet: Der Staat beobachtet seine Bürger, die Medien die Politiker, Ladenbesitzer ihre Kunden, Unternehmen ihre Arbeitnehmer, Erwachsene ihre Kinder und umgekehrt. Doch wehe dem, der unbeobachtet bleibt! Unbeobachtet zu bleiben bedeutet in einer Kommunikationsgesellschaft nicht wie in einer Disziplinar- und Kontrollgesellschaft verschont zu bleiben, sondern ausgeschlossen zu werden. Wer nicht beobachtet wird, kommt nicht vor, ist irrelevant. An die Stelle der Sichtbarkeitsfalle (vgl. Foucault 1977: 257) ist in der Kommunikations- und Mediengesellschaft die „Unsichtbarkeitsfalle“ (Schroer 2005: 333) getreten. Damit gehen wir von Feld 3 auf Feld 4 über:

\section{Feld 4) Irrelevanz: Unsichtbarkeit durch verweigerte Aufmerksamkeit der anderen}

Die vorübergehende Unaufmerksamkeit der Anderen (Feld 2) kann durchaus ein erwünschter Zustand sein. Wird sie zum Dauerzustand, wird aus dem Wunsch nach Anonymität ein handfester Ausschluss aus den Situationen reziproker Sichtverhältnisse. Insofern zieht eine sich verstetigende Aufmerksamkeitsverweigerung Exklusion nach sich, die sich auch räumlich niederschlagen kann - etwa in Banlieus, Slums und Favelas. Gerade an diesem Beispiel lässt sich noch einmal zeigen, dass wir es keineswegs mit einer umfassenden Visualisierungsoffensive zu tun haben, die sich zum Ziel gesetzt hat, jede Form von Unsichtbarkeit auszumerzen. Vielmehr ist festzustellen, dass jede Gesellschaft Bereiche kennt, die sie vor den Augen der Weltöffentlichkeit verbergen will. Während der Repräsentation dienende Orte durch ihre permanente Präsenz in den Medien ständig im Fokus der visuellen Aufmerksamkeit stehen, werden andere Regionen, Areale und Klientele systematisch unsichtbar gemacht. Für Beispiele sind Ausflüge in weit entfernte Regionen der Weltgesellschaft gar nicht nötig. Exklusion ereignet sich nicht nur in Bezug auf Randlagen und Außenseiter, sondern geht quer durch die Gesamtbevölkerung des Erdballs: „Für die Selbstdarstellung und Selbstfeier im Spektakel der Bilder ungeeignet erscheint der sexuell-unattraktive, alte, kranke und hässliche Körper; er erleidet Ausschluss und Ausgrenzung“" (Großklaus 2004: 33). Auch finden sich eine Vielzahl von Berufstätigen, deren Jobs sie zu unsichtbaren Dienern machen:

„Invisibility [...] is the most drastic form of attentiondeprivation, ultimately more painful and dehumanizing than hostile or other, negative' attention. Anyone can become temporarily invisible in meetings, groups, and other kinds of everyday situation. But for those whose jobs is regarded as dirty, unpleasant, or unsightly, and are therefore required to work in hidden places such as kitchens or basements, it is routine to their daily experience. Other workers of very low status, such as the cleaning lady or the busboy, while they may work in the purview of other people, remain invisible because others feel no need to acknowledge their presence." (Derber 2000: 70) 
Michael Herzfeld beschreibt in seinem Buch „The Social Production of Indifference“ (1992) die Fähigkeit von sozialen Institutionen, eine Mentalität zu schaffen, die die Aufgabe hat zu suggerieren, dass Außenseiter unsere Aufmerksamkeit nicht verdienen. Nicht zu übersehen ist allerdings, dass die dauerhafte Verweigerung der Aufmerksamkeit häufig einen Kampf um sie in Gang setzt. Zahlreiche politische Proteste, Aufstände und Revolten, aber auch Amokläufe, dienen dem Ziel, sich als von der Öffentlichkeit vernachlässigte Gruppe bzw. ignoriertes Individuum sichtbar zu machen. ${ }^{14}$ Dabei gilt:

„Je lauter man schreit, desto eher wird man wahrgenommen, um so gewisser ist also die eigene Existenz. Da die blasierte öffentliche Aufmerksamkeit sich von den immer zahlreicheren und grelleren Zerstreuungen gelangweilt zeigt, hat allein ein Schock, der stärker ist als die Schocks von gestern, eine Chance, die Aufmerksamkeit auf sich zu ziehen. [...] In unserer sensationssüchtigen Welt sind immer stärkere Reize notwendig, um die Aufmerksamkeit länger als einen flüchtigen Moment wachzuhalten. “ (Bauman 1996: 60)

Als Aufmerksamkeitsgeneratoren par excellence müssen dabei Gewalt und Terror gelten, denn gewalttätige Aktionen und terroristische Anschläge sind Handlungen, die garantiert nicht ignoriert werden, denn sie sind ,,auffällig“, und das heißt nach Auskunft des Bedeutungswörterbuch ,die Aufmerksamkeit auf sich ziehend, [...] unübersehbar“ (Duden 2002: 124). ${ }^{15}$ Wie unterschiedlich die einzelnen Akteure und Gruppen auch immer sein mögen, sie eint die Erfahrung, übersehen oder gar zum Verschwinden gebracht zu werden, und ihre Aktionen und Taten sind bestimmt von der Forderung, nicht länger übersehen, sondern endlich wahrgenommen zu werden, also von Feld 4 auf Feld 1 zu gelangen.

Mit diesem Modell wird der Anspruch erhoben, eine Vielzahl aktueller gesellschaftlicher Entwicklungen erfassen und analysieren zu können, nicht aber der, bereits eine umfassende Erklärung für sie bereitzuhalten. Angestrebt ist vielmehr, ein Instrumentarium anzubieten, mit dessen Hilfe die visuelle Kultur der gegenwärtigen Gesellschaft beschrieben werden kann, ohne dabei den Vereinseitigungen und Verkürzungen manch populärer Zeitdiagnose zu erliegen. Besonderer Wert wird dabei darauf gelegt, dass das Modell die auf und zwischen den Feldern herrschende Dynamik und die Möglichkeiten der Übergänge ebenso abzubilden in der Lage ist wie auch die Tendenz zur Verstetigung der sozialen Situationen zu relativ stabilen Lebenslagen. ${ }^{16}$ Ziel künftiger Unternehmungen könnte es sein, das Modell auf die verschie-

14 Was nicht heißen sollen, dass alle sozial unsichtbaren Gruppen ausnahmslos danach streben, gesehen zu werden, denn: „Mangelnde Beachtung kann [...] auch schonende Nischen schaffen.“ (Waldenfels 2002: 42) Und dies wissen nicht wenige Akteure durchaus zu schätzen.

15 Vgl. dazu Bruce Hoffman: „Es ist eine bekannte Tatsache, daß es dem Terrorismus ganz wesentlich darum geht, Aufmerksamkeit zu erregen und sich Publizität zu verschaffen, und daß selbst bei seinen frühesten Manifestationen vor Jahrhunderten die Zeloten und die Assasinen bewusst ein Publikum ansprachen, das weit über die unmittelbaren Opfer ihrer Angriffe hinausreichte." (Hoffman 1999: 178) Vgl. dazu auch Elter 2008.

16 Die Dynamik lässt sich etwa an folgendem Beispiel illustrieren: Ein Schüler hat einen besonders gelungenen Aufsatz verfasst und wird von seinem Deutschlehrer gebeten, nach vorne zu kommen und ihn vor der ganzen Klasse vorzutragen (Feld 1). In der darauf folgenden Mathematikstunde versucht sich derselbe Schüler vor den Blicken des Lehrers zu verstecken, da er seine Hausaufgabe nicht erledigt hat (Feld 2). Im sich anschließenden Englischunterricht wird eine Klassenarbeit geschrieben. Nach wiederholten Versuchen, bei seinem Nachbarn abzuschreiben, wird er an einen anderen Tisch gesetzt, wo ihn der Lehrer besser im Blick hat (Feld 3). In der letzten Stunde gelingt es unserem Schüler nicht, die Aufmerksamkeit seines Geschichtslehrers auf sich zu ziehen. Trotz wiederholten Aufzeigens ruft dieser ihn einfach nicht auf (Feld 4). Das Beispiel macht deutlich, dass es durchaus möglich ist, sich innerhalb eines (Schul-)Tages in allen vier Feldern zu bewegen. Daneben lassen sich jedoch auch Verhärtungen und Verfestigungen abbilden, die zu einem Verharren in einem der Felder führen, etwa wenn es dauerhaft nicht gelingt, der auch räumlich unterstützten Unsichtbarkeit zu entfliehen. 
densten sozialen Felder der Gesellschaft zu übertragen, um das Zusammenspiel von Selbstdarstellung, Kampf um Aufmerksamkeit, Sichtbarkeit / Unsichtbarkeit und visueller Kultur weiter aufzuschlüsseln.

\section{Literatur}

Anders, G. (1992): Die Antiquiertheit des Menschen. Bd. 2: Über die Zerstörung des Menschen im Zeitalter der dritten industriellen Revolution, München.

Arendt, H. (1979): Vom Leben des Geistes, München.

Balázs, B. (2001/1924): Der sichtbare Mensch oder die Kultur des Films, Frankfurt / Main.

Baudrillard, J. (1992): Transparenz des Bösen. Ein Essay über extreme Phänomene, Berlin.

Bauman, Z. (1995): Ansichten der Postmoderne, Hamburg.

Bauman, Z. (1996): Gewalt - modern und postmodern, in: M. Miller / H.-G. Soeffner (Hrsg.), Modernität und Barbarei. Soziologische Zeitdiagnosen am Ende des 20. Jahrhunderts, Frankfurt / Main, S. 36-67.

Beck, K. / W. Schweiger (Hrsg.) (2001): Attention please! Online-Kommunikation und Aufmerksamkeit, München.

Benjamin, W. (1972): Gewohnheit und Aufmerksamkeit, in: Ders., Gesammelte Schriften. Bd. IV / 1, Frankfurt / Main, S. 407-408.

Benjamin, W. (1977): Das Kunstwerk im Zeitalter seiner technischen Reproduzierbarkeit, in: Ders., Illuminationen, Frankfurt / Main, S. 136-169.

Berger, J. (1974): Sehen. Das Bild der Welt in der Bilderwelt, Reinbek / Hamburg.

Berger, P.L. (1977): Einladung zur Soziologie, München.

Bleicher, J.K. / K. Hickethier (Hrsg.) (2002): Aufmerksamkeit, Medien und Ökonomie, Münster - Hamburg - London.

Blumenberg, H. (2004): Zu den Sachen und zurück, Frankfurt / Main.

Blumenberg, H. (2006): Beschreibung des Menschen, Frankfurt / Main.

Boehm, G. (1994): Die Wiederkehr der Bilder, in: Ders. (Hrsg.), Was ist ein Bild?, München, S. 11-38.

Bolz, N. (2002): Das konsumistische Manifest, München.

Bourdieu, P. (1990): Was heißt sprechen? Die Ökonomie des sprachlichen Tausches, Wien.

Bourdieu, P. (1998): Über das Fernsehen, Frankfurt / Main.

Bourdieu, P. (2001): Das politische Feld. Zur Kritik der politischen Vernunft, Konstanz.

Bublitz, H. (2010): Im Beichtstuhl der Medien. Die Produktion des Selbst im öffentlichen Bekenntnis, Bielefeld.

Burri, R.V. (2008): Bilder als soziale Praxis: Grundlegungen einer Soziologie des Visuellen, in: Zeitschrift für Soziologie 37, S. 342-358.

Castells, M. (2002): Das Informationszeitalter. Bd. 2: Die Macht der Identität, Opladen.

Claessens, D. (1980): Das Konkrete und das Abstrakte. Soziologische Skizzen zur Anthropologie, Frankfurt / Main.

Crary, J. (2002): Aufmerksamkeit. Wahrnehmung und moderne Kultur, Frankfurt / Main.

Deleuze, G. (1992): Foucault, Frankfurt / Main.

Denzin, N. (1995): The Cinematic Society. The Voyeur's Gaze, London, S. 13-41.

Derber, C. (2000[1979]): The Pursuit of Attention. Power and Ego in Everyday Life, 2. Auflage, Oxford.

Duden (2002): Das Bedeutungswörterbuch, 3. neu bearbeitete und erweiterte Ausgabe, Mannheim - Leipzig - Wien - Zürich. 
Elter, A. (2008): Propaganda der Tat. Die RAF und die Medien, Frankfurt / Main.

Ernst, S. (1993): Schamlose Gesellschaft, in: G. Kneer / A. Nassehi / M. Schroer (Hrsg.), Soziologische Gesellschaftsbegriffe. Konzepte moderne Zeitdiagnosen, München, S. 51-75.

Foucault, M. (1973): Archäologie des Wissens, Frankfurt / Main.

Foucault, M. (1977): Überwachen und Strafen. Die Geburt des Gefängnisses, Frankfurt / Main.

Franck, G. (1998): Ökonomie der Aufmerksamkeit. Ein Entwurf, München - Wien.

Geisthövel, A. / H. Knoch (Hrsg.): Orte der Moderne. Erfahrungswelten des 19. und 20. Jahrhunderts, Frankfurt / Main - New York / NY.

Giddens, A. (1992): Die Konstitution der Gesellschaft. Grundzüge einer Theorie der Strukturierung, Frankfurt / Main - New York / NY.

Giddens, A. (1995): The Nation-State and Violence. Volume Two of A Contemporary Critique of Historical Materialism, Cambridge.

Goffman, E. (2009): Interaktion im öffentlichen Raum, Frankfurt / Main - New York / NY.

Großklaus, G. (2004): Medien-Bilder. Inszenierung der Sichtbarkeit, Frankfurt / Main.

Gumbrecht, H.U. (2001): 1926. Ein Jahr am Rande der Zeit, Frankfurt / Main.

Hahn, A. (2001): Aufmerksamkeit, in: A. Assmann / J. Assmann (Hrsg.), Aufmerksamkeiten. Archäologie der literarischen Kommunikation VII, München, S. 25-56.

Herzfeld, M. (1992): The Social Production of Indifference, Chicago / IL.

Hitzler, R. (1992): Der Goffmensch. Überlegungen zu einer dramatologischen Anthropologie, in: Soziale Welt 43, S. 449-461.

Hitzler, R. (1998): Das Problem, sich verständlich zu machen. Anthropologische Aspekte einer Dramatologie, in: H. Willems / M. Jurga (Hrsg.), Inszenierungsgesellschaft. Ein einführendes Handbuch, Opladen, S. 93-105.

Hitzler, R. (2007): Observation und Exhibition. Vom Leben im elektronischen Panoptikon, in: Sozialer Sinn. Zeitschrift für hermeneutische Sozialforschung 8, S. 385-391.

Hoffman, B. (2001): Terrorismus - der unerklärte Krieg. Neue Gefahren politischer Gewalt, Frankfurt / Main.

Holert, T. (2000): Bildfähigkeiten. Visuelle Kultur, Repäsentationskritik und Politik der Sichtbarkeit, in: Ders. (Hrsg.), Imageneering. Visuelle Kultur und Politik der Sichtbarkeit, Köln, S. 14-33.

Jenks, C. (1995): Visual Culture, London.

Jung, T. / S. Müller-Doohm (Hrsg.): Das Tabu, das Geheimnis und das Private, in: K. Imhof / P. Schulz (Hrsg.), Die Veröffentlichung des Privaten - die Privatisierung des Öffentlichen, Opladen, S. 136-146.

Kant, I. (2000): Anthropologie in pragmatischer Absicht, Hamburg.

Kirschner, J. (1999): So machen Sie auf sich aufmerksam. Fünfzehn Übungen, wie Sie aus Ihrem Schatten treten, München.

Knorr-Cetina, K. / U. Brügger (2005): Globale Mikrostrukturen der Weltgesellschaft. Die virtuellen Gesellschaften von Finanzmärkten, in: P. Windolf (Hrsg.), Finanzmarkt-Kapitalismus. Analysen zum Wandel von Produktionsregimen, Wiesbaden, S. 145-172.

Lasch, C. (1995): Das Zeitalter des Narzissmus, Hamburg.

Luhmann, N. (1983): Rechtssoziologie, 2. erweiterte Auflage, Opladen.

Luhmann, N. / P. Fuchs (1989): Reden und Schweigen, Frankfurt / Main.

Maar, C. / H. Burda (Hrsg.) (2004): Iconic Turn. Die neue Macht der Bilder, Köln.

Maase, K. (1997): Grenzenloses Vergnügen. Der Aufstieg der Massenkultur 1850-1970, Frankfurt / Main.

Mathiesen, T. (1997): Synopticon - The Viewer Society: Michel Foucault's 'Panopticon' Revisited, in: Theoretical Criminology 1, S. 215-234. 
Meyer, T. / M. Kampmann (1998): Politik als Theater. Die neue Macht der Darstellungskunst, Berlin.

Mead, G.H. (1968): Geist, Identität und Gesellschaft, Frankfurt / Main.

Mirzoeff, N. (1999): An Introduction to Visual Culture, London.

Mitchell, W.J.T. (2008 a): Pictorial Turn (1992), in: Ders., Bildtheorie, Frankfurt / Main, S. 101-135.

Mitchell, W.J.T. (2008 b): Das Leben der Bilder? Eine Theorie der visuellen Kultur. Mit einem Vorwort von Hans Belting, München.

Münch, R. (1991): Dialektik der Kommunikationsgesellschaft, Frankfurt / Main.

Münch, R. (1995): Dynamik der Kommunikationsgesellschaft, Frankfurt / Main.

Münsterberg, H. (1996): Das Lichtspiel. Eine psychologische Studie (1916) und andere Schriften zum Kino, Wien.

Neumann, O. (1971): Aufmerksamkeit, in: J. Ritter / K. Gründer (Hrsg.), Historisches Wörterbuch der Philosophie, Bd. 1, Basel, S. 635-645.

Neumann-Braun, K. (Hrsg.) (2011): Freundschaft und Gemeinschaft im Social Web. Bildbezogenes Handeln und Peergroup-Kommunikation auf Facebook \& Co, Baden-Baden.

Nolte, C. (2005): Kampf um Aufmerksamkeit. Wie Medien, Wirtschaft und Politik um eine knappe Ressource ringen, Frankfurt / Main - New York / NY.

Norman, D. A. (1973): Aufmerksamkeit und Gedächtnis. Eine Einführung in die menschliche Informationsverarbeitung, Weinheim - Basel.

Plessner, H. (2003): Grenzen der Gemeinschaft. Eine Kritik des sozialen Radikalismus (1924), in: Ders., Macht und menschliche Natur. Gesammelte Schriften, Bd. V, Frankfurt / Main, S. 7-133.

Pörksen, B. / W. Krischke (Hrsg.): Die Casting-Gesellschaft. Die Sucht nach Aufmerksamkeit und das Tribunal der Medien, Köln.

Raab, J. (2008): Visuelle Wissenssoziologie. Theoretische Konzeption und materiale Analysen, Konstanz.

Reichert, R. (2008): Amateure im Netz. Selbstmanagement und Wissenstechnik im Web 2.0, Bielefeld.

Ribot, T. (1908): Die Psychologie der Aufmerksamkeit, Leipzig.

Rifkin, J. (2000): Ein neuer Kapitalismus, in: Ders., Access. Das Verschwinden des Eigentums, Frankfurt / Main, S. 184-225.

Rössler, B. (2001): Der Wert des Privaten, Frankfurt / Main.

Rötzer, F. (1998): Digitale Weltentwürfe. Streifzüge durch die Netzkultur, München.

Säger, D. (2009): Nie wieder unsichtbar. Lieber mutig Profil zeigen als gar keinen Eindruck hinterlassen, München.

Scheler, M. (1954): Der Formalismus in der Ethik und die materiale Wertethik. Neuer Versuch der Grundlegung eines ethischen Personalismus, Bern.

Schroer, M. (2003): Sehen und Gesehen-werden. Von der Angst vor Überwachung zur Lust an der Beobachtung?, in: Merkur 57, S. 169-173.

Schroer, M. (2005): Sehen, Beobachten, Überwachen. Beitrag zu einer Theorie der Aufmerksamkeit, in: L. Hempel / J. Metelmann (Hrsg.), Bild - Raum - und Kontrolle. Videoüberwachung als Zeichen gesellschaftlichen Wandels, Frankfurt / Main, S. 325-341.

Schroer, M. (2007): Defizitäre Reziprozität. Der Raum der Überflüssigen und ihr Kampf um Aufmerksamkeit, in: C. Klinger / G.-A. Knapp / B. Sauer (Hrsg.), Achsen der Ungleichheit. Zum Verhältnis von Klasse, Geschlecht und Ethnizität, Frankfurt / Main, S. 257-270.

Schroer, M. (2010): Der Star, in: S. Moebius / M. Schroer (Hrsg.), Diven, Hacker, Spekulanten. Sozialfiguren der Gegenwart, Frankfurt / Main, S. 381-395.

Schroer, M. (2012): Gefilmte Gesellschaft. Beitrag zu einer Soziologie des Visuellen, in: C. Heinze / S. Moebius / D. Reicher (Hrsg.), Perspektiven der Filmsoziologie, Konstanz, S. 15-40. 
Schütz, A. / T. Luckmann (2003): Strukturen der Lebenswelt, Konstanz.

Seitter, W. (2001): Aufmerksamkeitskorrelate auf der Eben der Erscheinungen, in: A. Assmann / J. Assmann (Hrsg.), Aufmerksamkeiten. Archäologie der literarischen Kommunikation VII, München, S. 171-182.

Serres, M. (2005): Atlas, Berlin.

Serres, M. (1991ff): Hermes 1: Die Kommunikation, Berlin.

Sennett, R. (1983): Verfall und Ende des öffentlichen Lebens. Die Tyrannei der Intimität, Frankfurt / Main.

Simmel, G. (1992): Soziologie. Untersuchungen über die Formen der Vergesellschaftung, Gesammelte Werke Band 11, Frankfurt / Main.

Simmel, G. (1996): Der Begriff und die Tragödie der Kultur, in: Ders., Hauptprobleme der Philosophie. Philosophische Kultur. Gesamtausgabe Bd. 14, Frankfurt / Main, S. 385-416.

Soeffner, H.-G. (2004): Authentizitätsfallen und mediale Verspiegelungen. Inszenierungen im 20. Jahrhundert, in: Ders., Auslegung des Alltags - Der Alltag der Auslegung, Konstanz, S. 285-300.

Sofsky, W. (2007): Verteidigung des Privaten, Frankfurt / Main.

Sombart, W. (1988): Der Bourgeois. Zur Geistesgeschichte des modernen Wirtschaftsmenschen, Reinbek / Hamburg.

Spiegel (2/1993): Die schamlose Gesellschaft. Das Geschäft mit Sex und Gewalt, Ausgabe Nr. 47.

Stiegler, B. (2008): Die Logik der Sorge. Verlust der Aufklärung durch Technik und Medien, Frankfurt / Main.

Süddeutsche Zeitung (24.5.2004): Endloser Krieg, endloser Strom von Fotos. Die Folter der Gefangenen ist keine simple Verfehlung, Bericht von S. Sontag, Ausgabe Nr. 118, S. 13.

Thomas, W.S. (1965): Person und Sozialverhalten, Darmstadt.

Türcke, C. (2002): Die erregte Gesellschaft. Philosophie der Sensation, München.

Vattimo, G. (1992): Die transparente Gesellschaft, Wien.

Virilio, P. (1989): Die Sehmaschine, Berlin.

Waldenfels, B. (2004): Phänomenologie der Aufmerksamkeit, Frankfurt / Main.

Welsch, W. (1990): Zur Aktualität ästhetischen Denkens, in: Ders., Ästhetisches Denken, Stuttgart, S. 41-78.

Willems, H. (1998): Inszenierungsgesellschaft? Zum Theater als Modell, zur Theatralität der Praxis, in: H. Willems / M. Jurga (Hrsg.), Inszenierungsgesellschaft. Ein einführendes Handbuch, Opladen / Wiesbaden, S. 23-79.

ZEIT (27.4.2000): Versuch über die Intimität. Von Ballermann bis zu „Big Brother“, vom Internet bis zur Talkshow: Der neue Exhibitionismus grassiert, Bericht von U. Greiner, Ausgabe Nr. 18, S. 43.

Žižek, S. (2002): Kulturkapitalismus, in: Ders., Die Revolution steht bevor. Dreizehn Versuche über Lenin, Frankfurt / Main, S. 117-126.

Prof. Dr. Markus Schroer Philipps-Universität Marburg Institut für Soziologie Ketzerbach 11 35032 Marburg schroer@staff.uni-marburg.de 